\title{
Design Pitfalls lowering Acceptance of Technology-Based Interventions to Advocate Stair-Climbing
}

\author{
E. Beck \\ OFFIS Institute for Information \\ Technology \\ Germany \\ elke.beck@offis.de
}

\author{
D. Gansefort \\ Leibniz Institute for Prevention and \\ Epidemiology - BIPS \\ Germany \\ gansefort@leibniz-bips.de
}

\author{
S. Boll \\ University of Oldenburg \\ Germany \\ susanne.boll@uni-oldenburg.de
}

\author{
K. von Holdt \\ OFFIS Institute for Information \\ Technology \\ Germany \\ kai.von.holdt@offis.de \\ R. Malaka
University of Bremen
Germany
malaka@informatik.uni-bremen.de
}

\author{
L. Touma
University of Bremen
Germany
leen.tuma@uni-bremen.de \\ L. Touma
University of Bremen
Germany
leen.tuma@uni-bremen.de \\ L. Touma
University of Bremen
Germany
leen.tuma@uni-bremen.de \\ L. Touma
University of Bremen
Germany
leen.tuma@uni-bremen.de
}

J. Meyer
OFFIS Institute for Information
Technology
Germany
meyer@offis.de

\begin{abstract}
Stair climbing is a physical activity that can easily be performed in daily life and has a positive influence on health. We investigated the role of technology to promote stair climbing by designing and implementing two prototypes in real-life circumstances. To understand user acceptance and interaction we conducted user studies in a shopping mall and a university building. Based on our findings we derived lessons learned for designing future technology for the promotion of stair climbing.
\end{abstract}

\section{CCS CONCEPTS}

- Social and professional topics Socio-technical systems

\section{KEYWORDS}

Physical activity, stair climbing, prevention, interactive stairs, acceptance

\footnotetext{
ACM Reference format:

E. Beck, K. von Holdt, L. Touma, D. Gansefort, R. Malaka, J. Meyer, and S. Boll. 2019. Design Pitfalls lowering Acceptance of Technology-Based Interventions to Advocate Stair-Climbing. In Proceedings of ACM Pervasive Health conference, Trento, Italy, May 2019 (PervasiveHealth'19), 4

Permission to make digital or hard copies of part or all of this work for personal or classroom use is granted without fee provided that copies are not made or distributed for profit or commercial advantage and that copies bear this notice and the full citation on the first page. Copyrights for third-party components of this work must be honored. For all other uses, contact the owner/author(s). WOODSTOCK'18, Fune, 2018, El Paso, Texas USA

(c) 2018 Copyright held by the owner/author(s). 978-1-4503-0000-0/18/06...\$15.00 https://doi.org/10.1145/1234567890
}

pages. https://doi.org/10.1145/1234567890

\section{Introduction}

Lifelong physical activity is one of the key determinants of healthy aging and could delay or even prevent age-related frailty. However, globally, 1 out of 4 adults $(18+$ years $)$ is insufficiently active [18]. Stair climbing is advocated as one simple yet highly effective intervention to prevent frailty and improve cardiovascular fitness [11]. Thus, various health campaigns promote stair climbing using e.g. motivational signs [3] or banners [4].

As part of a long-term research project, we aim at investigating the role of sensors and interactive technology to promote stair climbing. We designed and technically implemented two interventions, the ActiStairs prototype and the InteractiveStairs prototype. We conducted small-scale, explorative user studies for each prototype to know whether they have a potential to get accepted by users, how they will probably be perceived and understood by users. The detailed findings from these studies are reported in [7] and [17]. Within this paper, we want to focus on some user acceptance-affecting pitfalls in the design of technology-based stair-climbing interventions, which we identified in the user studies, and discuss what we have learned from these.

\section{Related Work}

Some work exists that uses sensors for identifying stair climbing activity and interactive technology to provide motivating feedback to people. One approach is to use gamification and to 
direct attention towards the stairs via playful interaction with a staircase installation. For instance, Alhadidi et al. [1] present a sophisticated installation deploying sound and light displays, and relying on a local RFID infrastructure to provide personalized feedback in an interactive staircase installation. The Piano Stairs [16] motivate stair climbing by using a piano keyboard layout on the stairs to play sounds and enable persons to make music by going up and down the stairs. Peeters et al. [9] build on the Piano Stairs, identifying social collaboration as a motivating factor for stair climbing. Their design uses acoustics as a motivating and playful feedback. Rogers et al. [12] suggest a light-based intervention in a workplace setting; they found that for users decoding the meaning of the lights is difficult. Ciman et al. [2] developed a serious game for smartphones to incentivize people to use stairs instead of elevators or escalators. It uses smartphone sensors to count taken stair steps, in order to reach the top of real buildings, e.g. the Eiffel tower, within the game.

Another approach is to provide motivational messages or behavior-related information. The Apstairs system by Sakai et al. [13] uses Bluetooth scanning for detecting the presence of people's smartphones in order to present personalized motivational messages to encourage stair climbing in an office building. Two other prototypes by Rogers et al. [12] use abstract representations and diagrams to display elevator versus stair use. On the individual level various examples exist that motivate stair climbing as part of daily PA. For instance, activity trackers and smartphone apps monitor stair climbing and provide statistics.

\section{Prototype Design and Evaluation}

We designed and implemented two interventions, the ActiStairs prototype and the InteractiveStairs prototype, which explore different ways to promote stair climbing. While the ActiStairs prototype uses a playful approach for the general public in a semi-public setting, the InteractiveStairs prototype follows an individual-level approach with a focus on older adults as users.

\subsection{ActiStairs Prototype}

3.1.1 Concept and technical implementation

Our design objectives for ActiStairs were to use interactive technology to 1) get initial attention and motivate people to take the stairs, 2) give a reward to those who actually climb the stairs, and 3) provide additional information for those who are interested. The ActiStairs prototype consists of a 5m LED-Strip with a sensor unit, both placed on the left side of the stairs, and a monitor right at the foot of the stairs (Fig. 1, left). To get initial attention and motivate people to take the stairs, multiple blue lights on the LED-Strip are slowly crawling to the top, as an electronic analogy of footprint stickers [6]. A sensor unit (Raspberry Pi 3, two passive infrared (PIR) motion sensors) at the base of the stairs senses whenever someone takes the stairs up and triggers a red light pattern (Fig. 1, right), which accompanies the stair-climbing person. It runs for a predefined period of time before returning to the crawling blue lights and should act as a reward for taking the stairs. Moreover, each stair-climbing person contributes 30 meters to the joint goal of achieving a marathon run, building on top of the "Mount Everest" analogy of Eves [4]. This concept is visualized on a monitor next to the stairs, by providing an image of the marathon route and the current marathon's progress (Fig. 2). To keep up the motivation after the first finished marathon, the time needed to finish every marathon was measured and displayed as well. Finally, the monitor provides information about the health benefits of stair climbing and about the study itself.

\subsubsection{Evaluation Process}

We installed ActiStairs for 14 days at a flight of stairs in a shopping mall of a city with about 500.000 inhabitants. To understand people's reactions to and interaction with ActiStairs we inconspicuously observed 358 persons passing by or going up or down the stairs at three days during the two weeks for a total of five hours. Moreover, two researchers approached 20 of the observed persons for a 5-minutes interview on intervention acceptability, querying a person's perceptions of and attitudes towards ActiStairs, as well as personal characteristics (age, level of physical activity). Based on the acceptability of healthcare interventions framework by Sekhon et al. [14] we developed a 5item rating scale with (mainly) adjective pairs to assess a person's feeling about the intervention; the perceived amount of effort for participating; the intervention's fit with individual value system; understanding the intervention; benefits, profits, values that must be given up to engage; the perceived likeliness of the intervention to achieve purpose; and the participant's confidence that the required behavior can be performed.

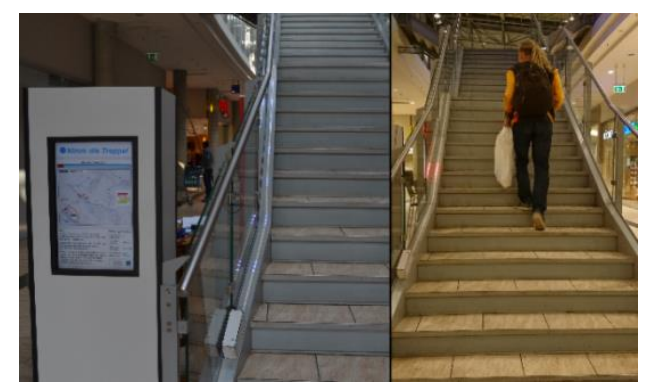

Figure 1: Left: entire installation of the intervention (monitor, sensor unit and LED-strip); Right: red light accompanying a person

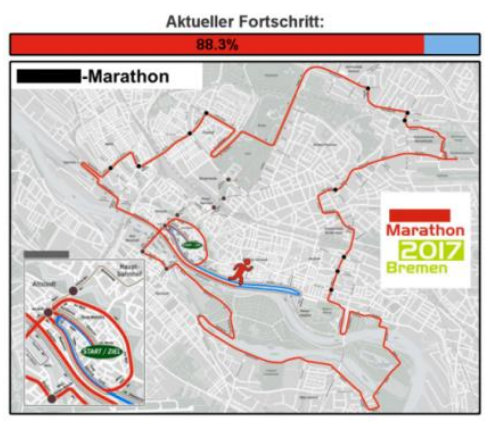

Figure 2: The marathon analogy used in the monitor display 


\subsubsection{Evaluation Results}

Overall, ActiStairs tends to be in line with people's value system. The majority of interviewed persons considered stair climbing as a useful preventative exercise, with a low burden and low opportunity costs for participating in stair climbing. The confidence to be able to participate was high. Only a minority of persons (two out of 19 respondents) saw disadvantages, finding it rather exhausting or impossible.

However, the interviewed persons differed in their perceptions of intervention coherence and perceived effectiveness. About half of the respondents tended to doubt the effectiveness, found it somewhat complicated and confusing. According to the observation, $22.6 \%$ of the observed persons noticed the intervention, $7.3 \%$ engaged more deeply.

The interviews further revealed some issues with noticing and understanding ActiStairs. Ten persons found that the overall ActiStairs system, particularly the light strip, was too unobtrusive to get noticed. Some suggested that the visual appearance should be more eye-catching or playful, but also commented that too much effects might annoy persons.

Seven persons mentioned that ActiStairs does not motivate, while three persons explicitly found it motivating. Some suggested getting better rewards and feedback after climbing the stairs. Thus, people did not always understand the display with the joint marathon on the base of the stairs as this reward and once even misinterpreted it as a marathon run commercial. Four persons had problems in understanding what the light strip is about, such as misunderstanding it as escape guidance.

\subsubsection{Lessons Learned}

Ensure sufficient perceptibility in the setting, but keep it unobtrusive: We observed display blindness in our setting, i.e. people ignoring or not even recognizing the intervention display. Moreover, the minimalistic LED strip may have been too small and not bright or colorful enough. Therefore, other means of visualization are worth investigating such as stair-mounted lights, similar to the stair riser banners that have been found to be effective [8].

Communicate health messages and rewards as explicitly as possible: The light strip in our intervention was quite abstract, and people had difficulties understanding what it was about, misunderstanding it e.g. as an emergency exit guide. It therefore is necessary to find presentations better reflecting the key message of the intervention about health and activity, technically implementing van Hoecke's finding [5] that health related messages are more effective than general ones. This should also include giving a reward to the stair-climbing person. In our case, not everybody understood the red light as such a reward, and our marathon display was positioned at the foot of the stairs, i.e. before the user performed the activity, and provided information but not a reward. A better way would be to place such a display at the top of the stairs, where the user might e.g. be applauded for contributing to the marathon.

\subsection{InteractiveStairs Prototype}

3.1.1 Concept and technical implementation
The InteractiveStairs prototype intends to motivate older adults aged between 65 and 75 years to use the stairs instead of taking the elevator through identifying and rewarding stair climbing behavior and informing about recommendations and health benefits. Its basic setup on one flight of stairs consists of two Bluetooth beacons (Estimote), one placed at the bottom and one at the top of the stairs (Fig. 3). Whenever a person is getting close to the stairs, the InteractiveStairs app on the person's smartphone identifies via the two beacons if the person is further going up the stairs. If the person approaches the top of the stairs, the app provides a sound notification as kind of immediate, direct feedback which plays an important role as a motivator and reminder that the person has something achieved. Moreover, as depicted in Fig. 4, the app provides information about the daily and weekly progress of climbed steps, a recommendation for weekly steps and the amount of obtained nanolives. This unit is based on the Microlife concept [15], which
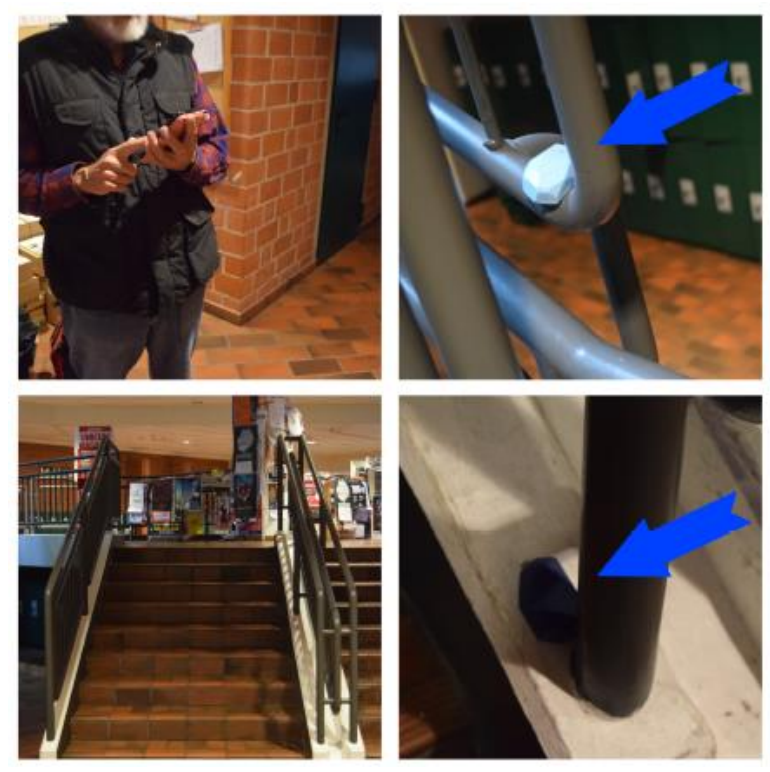

Figure 3: InteractiveStairs installation with two Bluetooth beacons at the top and bottom of stairs
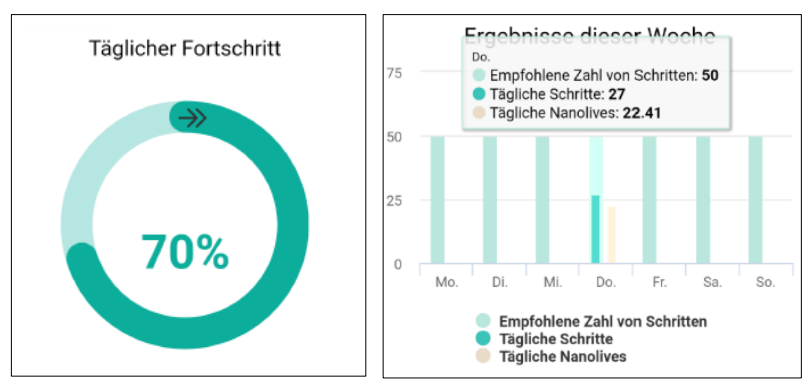

Figure 4: App screen with daily and weekly progress (recommended and actual steps on stairs, nanolives) 
gives healthy behavior a numerical expression. This should allow the person to take an informed decision about the amount of stair climbing.

\subsubsection{Evaluation Process}

The final InteractiveStairs prototype was the result of a longterm user-centered design process, involving a questionnaire and interview to inquire user preferences and needs, evaluations of mock-ups, and app interface evaluations. The evaluation of the final version of the prototype took place in the main hallway to the university cafeteria. Eight older adults (5 female, 3 male) aged between 65 and 75 years old participated in the evaluation study, which used hands-on demo, interview, questionnaire and observation to understand to which degree users initially accept and trust the prototype and its underlying concept.

\subsubsection{Evaluation Results}

Most study participants considered the InteractiveStairs prototype as trustworthy and easy to use, and the underlying concept as easy to understand. Overall, 6 out of 8 people understood the prototype very well, knew the meaning of the sound notification and the microlife concept in context of the prototype. Similarly, most participants found the prototype useful. However, when asked about negative aspects of the prototype that affect its acceptance, one person found that the prototype neglected considering other forms of physical activity, which contribute to a healthy life too. Three persons mentioned that the prototype did not show any information about breathing. They see this as an important point to be presented because while going up the stairs breathing can get relatively difficult, especially, if the person has asthma. Another point which was mentioned by three people is that having a smart phone is a condition here. No matter where the smart phone is put, some did not like the idea of having it always with them. One person suggested using a wrist-worn device instead of a smartphone.

\subsubsection{Lessons Learned}

Add functionality to increase overall usefulness of the intervention: Albeit striving for simple functionality to aid the ease of use of the prototype, adding further functionality such as integrating biometric data (e.g. breathing rate) and data on other physical activity might improve the usefulness of the intervention. The prototype could act as a microlife wallet, which saves all microlives from many health applications on the smartphone.

Consider ambient and body-worn technologies for immediate rewarding health-related feedback: Despite increasing levels of smartphone use in populations aged 65 years and above [10], low-intensity use patterns, such as not taking a smart phone along all the time, still persist. This suggests to rather using ambient or body-worn technologies for immediately rewarding health-supporting behavior.

\section{Conclusions and Outlook}

We designed and evaluated two technology-based interventions to encourage stair climbing. The user studies provided insights into design issues that affect user interaction with and acceptability of such an intervention. We found that technology clearly is an interesting tool that can contribute to increasing awareness for stair climbing, as long as it masters the delicate balance between perceptibility and unobtrusiveness, as well as usefulness and comprehension of the intervention. Based on our findings, future work could further investigate the effectiveness of certain designs for specific target groups, also taking into account the intervention's physical context.

\section{ACKNOWLEDGMENTS}

The study was funded by the German Federal Ministry of Education and Research (Project No.'s 01EL1422C, 01EL1422A).

\section{REFERENCES}

[1] S. Alhadidi, J. McLean, L. Sharah, I. Chia, and R. Sam. 2016. Multiflight Creating Interactive Stairs through Positive Technology. In 8th Arab Sociecty for Computer Aided Architectural Design (ASCAAD) Conference, 395-998.

[2] M. Ciman, M. Donini, O. Gaggi, and F. Aiolli. 2016. Stairstep Recognition and Counting in a Serious Game for Increasing Users' Physical Activity. Personal Ubiquitous Comput. 20, 6, 1015-1033.

[3] M. S. Dolan, L. A. Weiss, R. A. Lewis, A. Pietrobelli, M. Heo, and M. S. Faith. 2006. "Take the stairs instead of the escalator": effect of environmental prompts on community stair use and implications for a national "Small Steps" campaign. Obesity reviews 7, 1, 25-32.

[4] F. F. Eves, E. K. Olander, G. Nicoll, A. Puig-Ribera, and C. Griffin. 2009. Increasing stair climbing in a train station: The effects of contextual variables and visibility. Journal of Environmental Psychology 29, 2, 300-303.

[5] A.-S. Van Hoecke, J. Seghers, and F. Boen. 2018. Promoting Stair Climbing in a Worksite and Public Setting: Are Footprints Enough? American Journal of Health Promotion 0, 0, 890117117694284

[6] A. L. Lewis and F. F. Eves. 2012. Prompts to Increase Stair Climbing in Stations: The Effect of Message Complexity. Journal of Physical Activity and Health 9, 7, 954-961.

[7] J. Meyer, E. Beck, K. von Holdt, D. Gansefort, T. Brand, H. Zeeb, and S. Boll. 2018. ActiStairs: Design and Acceptance of a Technology-Based Intervention to Advocate Stair-Climbing in Public Spaces. In Proc. of the 3rd Int. Workshop on Multimedia for Personal Health and Health Care, 59-66.

[8] M. Nocon, F. Müller-Riemenschneider, K. Nitzschke, and S. N. Willich. 2010. Review Article: Increasing physical activity with point-of-choice prompts - a systematic review. Scandinavian Journal of Public Health 38, 6, 633-638.

[9] M. Peeters, C. Megens, E. van den Hoven, C. Hummels, and A. Brombacher. 2013. Social Stairs: Taking the Piano Staircase towards Long-Term Behavioral Change. In Proc. of the Int. Conference on Persuasive Technology, 174-179.

[10] Pew Research Center. 2017. "Tech Adoption Climbs Among Older Adults"

[11] W. J. Rejeski, R. Axtell, R. Fielding, J. Katula, A. C. King, T. M. Manini, A. P. Marsh, M. Pahor, A. Rego, C. Tudor-Locke, and others. 2013. Promoting physical activity for elders with compromised function: the lifestyle interventions and independence for elders (LIFE) study physical activity intervention. Clinical interventions in aging 8, 1119.29

[12] Y. Rogers, W. R. Hazlewood, P. Marshall, N. Dalton, and S. Hertrich. 2010. Ambient Influence: Can Twinkly Lights Lure and Abstract Representations Trigger Behavioral Change? In Proc. of the 12th ACM Int. Conference on Ubiquitous Computing, 261-270.

[13] R. Sakai, S. Van Peteghem, L. van de Sande, Peter Banach, and Maurits Kaptein. 2011. Personalized Persuasion in Ambient Intelligence: The APStairs System. In Proc. of the 2nd International Joint Conference on Ambient Intelligence 2011, Springer, Berlin, Heidelberg, 205-209.

[14] M. Sekhon, M. Cartwright, and J. J. Francis. 2017. Acceptability of healthcare interventions: an overview of reviews and development of a theoretical framework. BMC Health Services Research 17, 1, 88.

[15] D. Spiegelhalter. 2012. Using speed of ageing and "microlives" to communicate the effects of lifetime habits and environment. BMJ: British Medical Journal (Online), 345.

[16] The Fun Theory. 2009. Piano Staircase.

[17] L. Touma. 2019. Interactive Stairs: Using Sensors and the Microlife Concept to Encourage Seniors to Climb Stairs. Master's thesis. University of Bremen, Bremen, Germany.

[18] WHO. Prevalence of insufficient physical activity. https://www.who.int/gho/ncd/risk_factors/physical_activity_text/en/ 